\title{
El valor agregado bruto del Ecuador 2007-2017
}

\author{
The gross value added of Ecuador 2007-2017
}

\author{
Salinas Campuzano Davis Xavier* \\ Universidad Técnica de Machala \\ dsalinas3@utmachala.edu.ec \\ Machala - Ecuador \\ Cedeño Alonso Joselyn Ivonne \\ Universidad Técnica de Machala \\ jcedeno2@utmachala.edu.ec \\ Machala - Ecuador \\ Vega Jaramillo Flor Yelena \\ Universidad Técnica de Machala \\ fvega@utmachala.edu.ec \\ Machala - Ecuador

\section{Sotomayor Pereira Jorge Guido} \\ Universidad Técnica de Machala \\ jsotomayor@utmachala.edu.ec \\ Machala - Ecuador
}

\section{Resumen}

El presente trabajo tiene como objetivo analizar el valor agregado bruto del Ecuador para el periodo 2007-2017, que permita la descripción de las provincias con mayor y menor actividad económica. En este sentido, también se persigue la valoración del VAB de la provincia de El Oro y su relevancia para la economía regional y nacional. Para tal efecto se empleó una metodología de investigación de tipo documental, utilizando fuentes secundarias para la recopilación de información con el objetivo de analizar la variable objeto de estudio; además del método analítico-sintético e inductivo- deductivo para la elaboración del marco teórico. Los resultados del análisis evidencian que el VAB en Ecuador crece a una tasa promedio del $7,05 \%$ y las provincias con mayor aporte son Pichincha y Guayas con el 51,76\% del total, lo cual refleja la alta concentración de la producción en el espacio geográfico ecuatoriano (generación de una relación centroperiferia). En este sentido también se realiza una valoración del VAB de la provincia de El Oro desde la perspectiva de su evolución, composición, aporte de cada cantón al total y de cada sector económico.

Palabras clave: Crecimiento Económico, Valor Agregado Bruto, Sectores Económicos, Economía Ecuatoriana. 


\begin{abstract}
This work aims to analyze the gross added value of Ecuador for the period 2007-2017, which allows the description of the provinces with the highest and lowest economic activity. In this sense, the assessment of the GVA of the province of El Oro and its relevance for the regional and national economy is also pursued. For this purpose, a documentary-type research methodology was used, using secondary sources for the compilation of information in order to analyze the variable under study; in addition to the analytical-synthetic and inductive-deductive method for the elaboration of the theoretical framework. The results of the analysis show that the GVA in Ecuador grows at an average rate of $7.05 \%$ and the provinces with the highest contribution are Pichincha and Guayas with $51.76 \%$ of the total, which reflects the high concentration of production in the Ecuadorian geographic space (generation of a center-periphery relationship). In this sense, an assessment of the GVA of the province of El Oro is also carried out from the perspective of its evolution, composition, contribution of each canton to the total and of each economic sector.
\end{abstract}

Keywords: Economic Growth, Gross Value Added, Economic Sectors, Ecuadorian Economy.

\title{
Introducción
}

El Ecuador se ha caracterizado a lo largo de la historia como un país productor y exportador de materias primas y recursos naturales. Estas actividades productivas representan la columna vertebral del comercio internacional del país con el resto del mundo, constituyéndose en las principales fuentes de ingresos de divisas al Estado, y esto a su vez generando mayor crecimiento económico. El Producto Interno Bruto (PIB) es la suma de los valores agregados de las diversas etapas de producción y en todos los sectores de la economía. A partir del valor añadido bruto de un país se obtiene fácilmente el PIB ya que ambos guardan una estrecha relación. El PIB se obtiene después de añadirle al valor agregado del país, los valores por consumo intermedio. A nivel país, este agregado es el principal indicador macroeconómico que demuestra el crecimiento económico y el desarrollo productivo de bienes y servicios. La presente investigación analiza la evolución y características del Valor Agregado Bruto (VAB) ecuatoriano y de la provincia de El Oro para el periodo 2007-2017, empleando para el efecto el método histórico -lógico de tipo descriptivo a través de fuentes bibliográficas y estadísticas tales como: Banco Central del Ecuador (BCE), el analítico-sintético e inductivo-deductivo para la elaboración del marco teórico. Los resultados del análisis evidencian que el VAB en Ecuador crece a una tasa promedio del 7,05\% y las provincias con mayor aporte son Pichincha y Guayas con el 51,76\% del total, lo cual refleja la alta concentración de la producción en el espacio geográfico ecuatoriano (generación de una relación centro-periferia). En este sentido también se realiza una valoración del VAB de la provincia de El Oro desde la perspectiva de su evolución, composición, aporte de cada cantón al total y de cada sector económico. 


\section{El Valor Agregado Bruto en Ecuador: Periodo 2007 - 2017}

Según el Banco Mundial, el indicador macroeconómico que mide el valor añadido realizado por cada sector o industria en el proceso productivo de bienes y servicios finales, realizados en un periodo de tiempo determinado y en un área específica es el VAB. Este indicador sirve de base para la estimación del PIB. En Ecuador el VAB es considerado como uno de los indicadores de gran importancia con respecto a la evolución económica. El valor agregado bruto se lo considera como la fuente de la que provienen los ingresos primarios del sistema económico. (Zamora \& Coello, 2015)

El VAB es importante al momento de analizar el crecimiento económico dentro de un país, dado que (Pereira, Apunte, \& Pulgar, 2018) lo definen como el valor de la producción de la actividad económica menos el valor del consumo intermedio de la misma, siendo considerado como una medida que contribuye al PIB de un país. En definición económica establece la producción de una organización, y las compras de materias primas, suministros y servicios que son necesarios para obtener dicha producción. Es decir, se conceptualiza como el resultado final de la actividad de la producción de las unidades residentes y se corresponde con la producción de bienes y servicios de la economía (Guerra \& González, 2015). Se considera al VAB como la expresión del crecimiento económico, y se conceptualiza como el resultado final de toda actividad económica productiva en su conjunto. (Aravena \& Fuentes, 2013) establecen que el aumento o crecimiento del valor agregado se descompone tanto del cambio en las horas trabajadas y en la productividad de la mano de obra.

El cálculo del valor agregado bruto se realiza de la siguiente manera: se resta al valor de la producción total el de los bienes y servicios que representan el consumo intermedio, de esta manera, los bienes y servicios que fueron utilizados en la producción, al ser sometidos a transformación, adquieren un valor superior, esta diferencia recibe el nombre de valor agregado bruto (Vega, Brito, Apolo, \& Sotomayor, 2020).

$$
V A B=P G B=V B P-C I
$$

Donde, VBP es el Valor Bruto de la Producción y CI es el Consumo Intermedio de la unidad productiva considerada. El VAB puede ser determinado a precios de productor o a precios básicos. En la medición recoge el valor agregado de bienes y servicios relacionados a los ejes productivos. Asimismo, recopila datos relativos a las contribuciones finales en cada industria. El VAB en Ecuador ha mantenido un constante crecimiento, las provincias de mayor representatividad para el país son Pichincha y Guayas, destacándose las actividades del sector primario como eje principal.

Ecuador ha sido reconocido como uno de los países con mayores exportaciones de materia prima, goza de una economía abierta a nivel mundial, sin embargo, se ha visto afectado por diversos factores que han minado su estabilidad, lo cual ha generado alteraciones en el crecimiento económico productivo del mismo. En términos generales, en la década de los 70s Ecuador mantuvo una tasa de crecimiento de 2,20\%. A partir de 1965, el país presentó un crecimiento acelerado hasta mediados de los años 70, periodo que fue marcado por el boom petrolero. Los principales factores que explicaron el crecimiento en este periodo fueron el consumo y la formación bruta de capital (Brito, Sotomayor, \& Apolo, 2019).

En el año 1978 se originó un decrecimiento en la tasa de VAB debido a la falta de liquidez en el sector productivo, y en la caída de los precios del petróleo, principal recurso en la 
economía ecuatoriana. Entre 1982 y 1983 se dieron diversas reformas comerciales que impactaron en el conjunto de la economía. En los años 1997-1998, el Ecuador tuvo que enfrentar nuevas crisis; caída de los precios del petróleo y el desastre natural causado por el fenómeno de El Niño, los problemas se profundizaron, el precio del petróleo no se recuperó y el sistema financiero del país entró en crisis.

A partir del 2000, Ecuador recuperó su tasa de crecimiento promovida por el consumo y las exportaciones dando como resultado una relocalización estratégica del sector económico ecuatoriano. Desde este periodo, el país tuvo significativas variaciones en sus tasas de crecimiento, principalmente en el valor agregado bruto y otros indicadores indispensables para la economía ecuatoriana.

La tasa de crecimiento promedio entre los años 2000-2009 de 4,6\% (Brito, Sotomayor, \& Apolo, 2019) fue debido a que algunos sectores profundizaron su actividad económica de manera satisfactoria, el ajuste del tipo de cambio nominal originado en el país a principios del año 2000 promovió principalmente las exportaciones, generando paulatinamente aumentos directos al valor agregado bruto en Ecuador.

Las exportaciones, la inversión y el consumo han generado altas tasas de crecimiento en relación al VAB en Ecuador. Para el año 2007, la provincia de Orellana ocupaba los primeros puestos con el mayor valor agregado bruto; los siguientes años, estos puestos eran ocupados por Guayas y Pichincha, siendo éstas las que manejaron mejor su sector económico; y ocupando el último lugar, la provincia de Morona Santiago. Pichincha y Guayas presentaron mejores resultados en su VAB 2017, estas provincias, además de contar con un mayor volumen productivo, son las que tienen mayor concentración poblacional y se ubican entre las cinco mejores provincias que poseen mayores ingresos promedio en los hogares (García \& Ochoa, 2018).

Las industrias consideradas en la metodología del BCE para estimar el VAB son catorce: agricultura, ganadería, silvicultura y pesca; explotación de minas y canteras; manufactura; suministro de electricidad y de agua; construcción; comercio; actividades de alojamiento y de comidas; transporte, información y comunicaciones; actividades financieras; actividades profesionales e inmobiliarias; administración pública; enseñanza; salud y otros servicios. Cruz \& Polanco (2014) manifiestan que, a partir de la etapa de industrialización, el sector primario comenzó a contribuir al crecimiento económico del país, ya que, si en cada región de Ecuador se satisface la demanda de alimentos, también permite que el exceso de exportación aumente generando de la misma forma ingresos para el país, y a su vez identificando el crecimiento del VAB.

El BCE menciona que algunas actividades económicas crecieron debido a los impulsos realizados para aumentar el valor agregado bruto, el sector primario tanto como la agricultura, ganadería, silvicultura, pesca y explotación de minas ocupan los dos primeros puestos en relación al crecimiento del VAB en el periodo 2007 - 2017. 


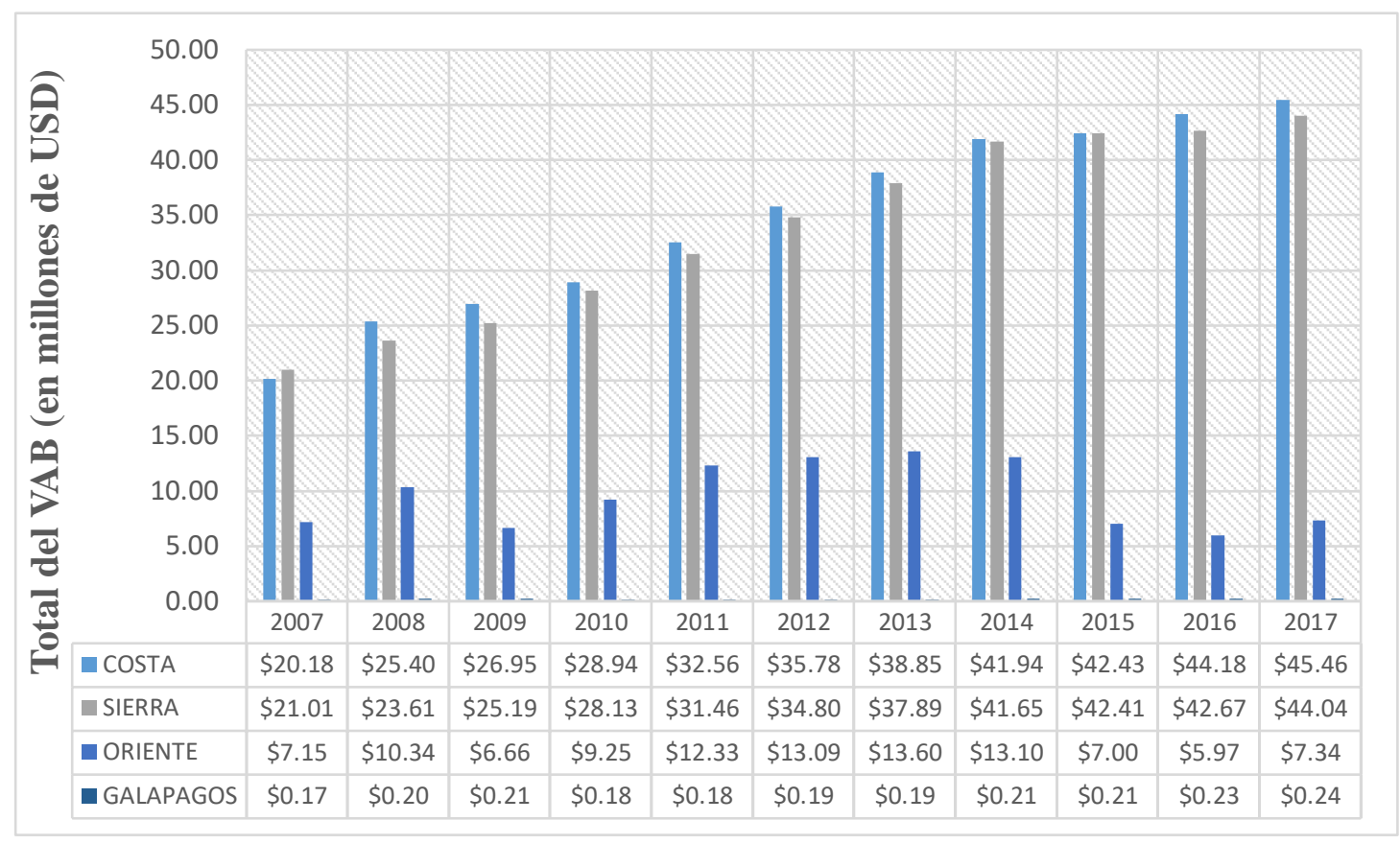

Figura 1. VAB por Regiones del Ecuador. Período 2007-2017 (en millones de USD). Fuente: Banco Central del Ecuador (2019).

En la Figura 1 se puede apreciar la última información oficial presentada por la Dirección de Estadística Económica del BCE, sobre las cuentas regionales desagregadas por cada uno de los cantones que tiene cada provincia en el Ecuador, el total del valor agregado es de USD 97.082 al año 2017, siendo la Región Costa una de las más generadoras de valor, con USD 45.4 millones, con una tasa promedio entre 2007 - 2017 de 8,64\%; mientras que la Región Sierra aportó con USD 44.035 millones y crece para el periodo anteriormente señalado a una tasa promedio del 7,76\%. La Región del Oriente tiene una tasa promedio de crecimiento del 4,94\% y aportó USD 7.300 millones, y por último la Región Insular o Galápagos que es la que menor aporte representa, con una tasa de crecimiento del 3,92\% y un aporte de USD 242.6 millones al VAB nacional.

\section{Materiales y métodos}

La metodología empleada en la investigación corresponde a una investigación de tipo documental. Para tal efecto se utilizaron datos secundarios como fuente de información, esto es, textos, artículos, libros e información estadística proveniente páginas oficiales, fundamentalmente del BCE. La información recopilada se sistematizó y se presentó en tablas y gráficos estadísticos explicativos de la variable objeto de estudio. Según (Gómez Luna et al, 2014) indican que este tipo de investigación garantiza que se obtenga la información más importante que permita el desarrollo del trabajo. Mientras que (Tancara Q., 1993) destaca que "la investigación documental, como una serie de métodos y técnicas de búsqueda, procesamiento y almacenamiento de la información contenida en los documentos, en primera instancia, y la presentación sistemática, coherente y 
suficientemente argumentada de nueva información en un documento científico" (pág.94). El proceso de investigación inició con la búsqueda de la información, para lo cual se accedió a gestores de información científica como Scielo, Redalyc, revista Universidad Nacional Autónoma de México (UNAM) y repositorio de la Universidad Técnica de Machala (UTMACH).

\section{Resultados y discusión}

El VAB es un indicador económico que muestra la diferencia entre la producción y el consumo intermedio en un periodo y área específica determinada. En este sentido mide el valor añadido realizado por cada sector o industria en el proceso de producción de bienes y servicios finales, sirviendo de base para la estimación del PIB.

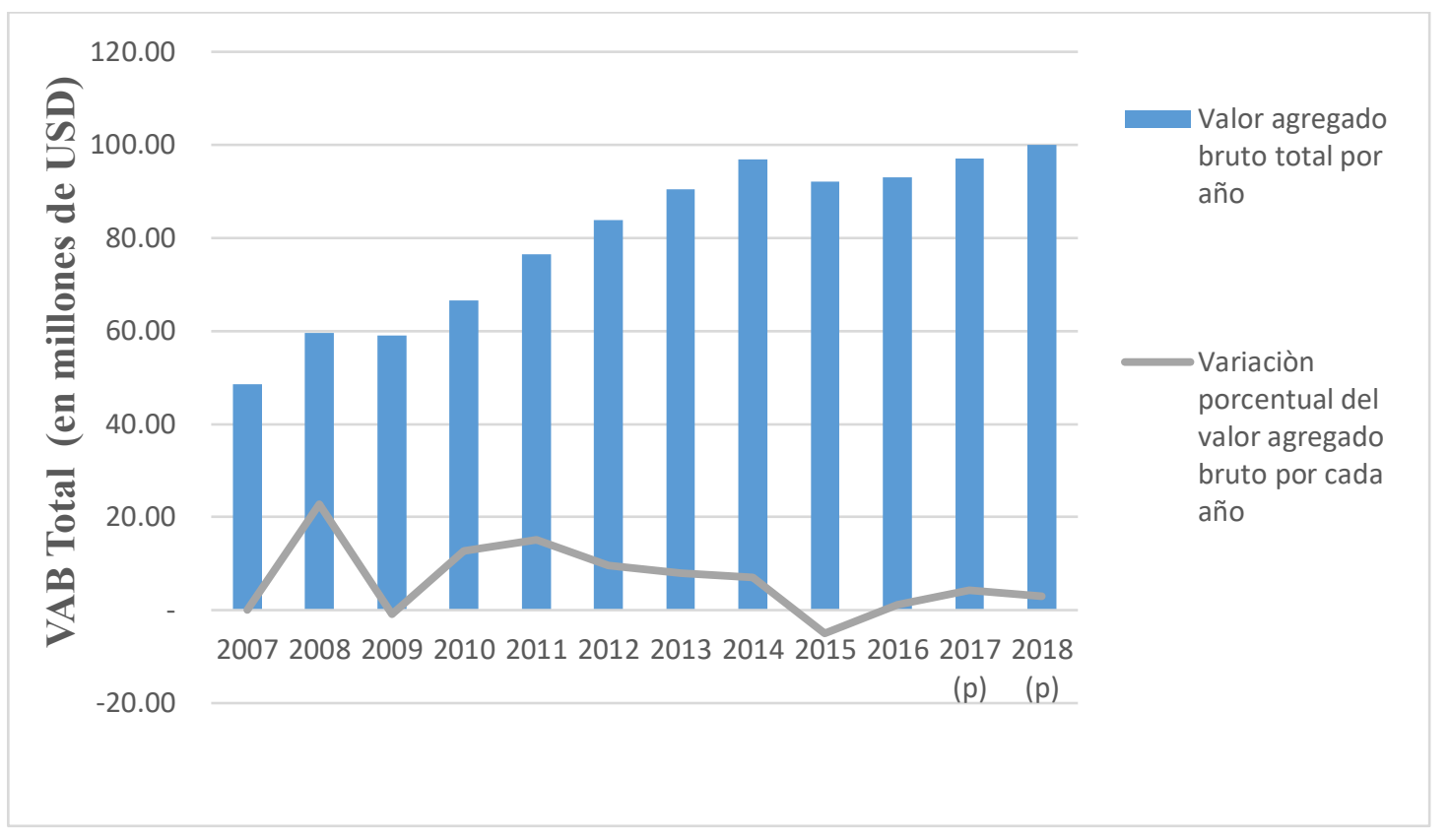

Figura 2. Valor Agregado Bruto Total del Ecuador. Período 2007 -2018

Fuente: Banco Central del Ecuador (2019) 
Tabla 1. Valor Agregado Bruto total. Periodo 2007 -2018

\begin{tabular}{|c|c|c|}
\hline Año & $\begin{array}{c}\text { Valor agregado bruto total por } \\
\text { año (USD) }\end{array}$ & $\begin{array}{c}\text { Variación porcentual } \\
\text { del valor agregado bruto } \\
\text { por cada año (\%) }\end{array}$ \\
\hline 2007 & 48.510 .903 & - \\
\hline 2008 & 59.550 .902 & 22,76 \\
\hline 2009 & 59.013 .275 & $-0,90$ \\
\hline 2010 & 66.499 .460 & 12,69 \\
\hline 2011 & 76.536 .215 & 15,09 \\
\hline 2012 & 83.856 .171 & 9,56 \\
\hline 2013 & 90.533 .862 & 7,96 \\
\hline 2014 & 96.894 .741 & 7,03 \\
\hline 2015 & 92.042 .505 & $-5,01$ \\
\hline 2016 & 93.038 .286 & 1,08 \\
\hline $2017(\mathrm{p})$ & 97.082 .733 & 4,35 \\
\hline $2018(\mathrm{p})$ & 99.955 .953 & 2,96 \\
\hline TOTAL & 963.515 .006 & 77,60 \\
\hline
\end{tabular}

Fuente: Banco Central del Ecuador (2019)

La Figura 2 refleja el crecimiento del VAB en Ecuador para el periodo 2007-2018, el cual muestra un crecimiento promedio del 7,05\% para el periodo señalado. En la Tabla 1 se evidencia los valores correspondientes de este periodo en el cual para el 2007 el VAB fue USD 48.510 millones, y para el 2018 de USD 99.995 millones. Si excluimos al año 2008 la tasa de crecimiento es del $5.48 \%$, esto debido a que en este año los altos precios del petróleo influyeron en la conformación del VAB, además del aumento de las inversiones públicas y privadas que ocasionó un crecimiento en la economía del país. En términos generales la tasa de crecimiento es bastante aceptable. A partir del 2012 se puede evidenciar un fenómeno contrario, debido a que hubo una contracción en el sector petrolero con la caída del precio de este producto. 
Recibido: 12 enero de 2021 / Aceptado: 05 de junio de 2021

Conference Proceedings UTMACH V.5 Núm.1 (2021)

ISSN: 2588-056X

Tabla 2. Valor Agregado Bruto Petrolero y no Petrolero del Ecuador. Periodo 2007 -2017 (en millones de USD).

\begin{tabular}{|c|c|c|c|c|c|c|c|c|c|c|c|c|}
\hline \multirow[t]{2}{*}{ Año } & \multicolumn{4}{|c|}{ Millones de dólares } & \multicolumn{4}{|c|}{ Millones de dólares de 2007} & \multicolumn{4}{|c|}{ Tasas de variación } \\
\hline & $\begin{array}{c}\text { Valor } \\
\text { agregado } \\
\text { petrolero }\end{array}$ & $\begin{array}{c}\text { Valor } \\
\text { agregado no } \\
\text { petrolero }\end{array}$ & $\begin{array}{l}\text { Otros } \\
\text { elementos } \\
\text { del PIB }\end{array}$ & P.I.B. & $\begin{array}{c}\text { Valor } \\
\text { agregado } \\
\text { petrolero }\end{array}$ & $\begin{array}{c}\text { Valor } \\
\text { agregado no } \\
\text { petrolero }\end{array}$ & $\begin{array}{l}\text { Otros } \\
\text { elementos } \\
\text { del PIB }\end{array}$ & P.I.B. & $\begin{array}{c}\text { Valor } \\
\text { agregado } \\
\text { petrolero }\end{array}$ & $\begin{array}{c}\text { Valor } \\
\text { agregado } \\
\text { no } \\
\text { petrolero }\end{array}$ & $\begin{array}{c}\text { Otros } \\
\text { elementos } \\
\text { del PIB }\end{array}$ & P.I.B. \\
\hline 2007 & $6.751 .274,0$ & $41.759 .629,0$ & $2.496 .874,0$ & $51.007 .777,0$ & $6.751 .274,0$ & $41.759 .629,0$ & $2.496 .874,0$ & $51.007 .777,0$ & $-7,1$ & 3,6 & 7,0 & 2,2 \\
\hline 2008 & $9.773 .162,0$ & $49.777 .740,0$ & $2.211 .733,0$ & $61.762 .635,0$ & $6.800 .916,0$ & $44.703 .118,0$ & $2.746 .374,0$ & $54.250 .408,0$ & 0,7 & 7,0 & 10,0 & 6,4 \\
\hline 2009 & $5.894 .526,0$ & $53.118 .749,0$ & $3.506 .411,0$ & $62.519 .686,0$ & $6.829 .481,0$ & $45.522 .029,0$ & $2.206 .222,0$ & $54.557 .732,0$ & 0,4 & 1,8 & $-19,7$ & 0,6 \\
\hline 2010 & $8.126 .685,0$ & $58.372 .775,0$ & $3.055 .907,0$ & $69.555 .367,0$ & $6.672 .076,0$ & $47.598 .618,0$ & $2.210 .361,0$ & $56.481 .055,0$ & $-2,3$ & 4,6 & 0,2 & 3,5 \\
\hline 2011 & $10.902 .229,0$ & $65.633 .986,0$ & $2.740 .449,0$ & $79.276 .664,0$ & $6.925 .325,0$ & $51.507 .763,0$ & $2.491 .976,0$ & $60.925 .064,0$ & 3,8 & 8,2 & 12,7 & 7,9 \\
\hline 2012 & $11.742 .367,0$ & $72.113 .804,0$ & $4.068 .373,0$ & $87.924 .544,0$ & $7.141 .540,0$ & $54.602 .990,0$ & $2.617 .903,0$ & $64.362 .433,0$ & 3,1 & 6,0 & 5,1 & 5,6 \\
\hline 2013 & $11.979 .019,0$ & $78.554 .843,0$ & $4.595 .797,0$ & $95.129 .659,0$ & $7.021 .754,0$ & $57.900 .337,0$ & $2.624 .037,0$ & $67.546 .128,0$ & $-1,7$ & 6,0 & 0,2 & 4,9 \\
\hline 2014 & $11.198 .789,0$ & $85.695 .952,0$ & $4.831 .590,0$ & $101.726 .331,0$ & $7.135 .471,0$ & $60.503 .777,0$ & $2.466 .114,0$ & $70.105 .362,0$ & 1,6 & 4,5 & $-6,0$ & 3,8 \\
\hline 2015 & $5.152 .475,0$ & $86.890 .030,0$ & $7.247 .876,0$ & $99.290 .381,0$ & $6.950 .966,0$ & $61.237 .760,0$ & $1.985 .951,0$ & $70.174 .677,0$ & $-2,6$ & 1,2 & $-19,5$ & 0,1 \\
\hline 2016 & $4.275 .171,0$ & $88.763 .115,0$ & $6.899 .410,0$ & $99.937 .696,0$ & $7.171 .149,0$ & $60.407 .935,0$ & $1.734 .982,0$ & $69.314 .066,0$ & 3,2 & $-1,4$ & $-12,6$ & $-1,2$ \\
\hline $\begin{array}{l}2017 \\
\text { (p) }\end{array}$ & $5.639 .977,0$ & $91.442 .756,0$ & $7.213 .129,0$ & $104.295 .862,0$ & $7.008 .523,0$ & $61.668 .088,0$ & $2.279 .080,0$ & $70.955 .691,0$ & $-2,3$ & 2,1 & 31,4 & 2,4 \\
\hline $\begin{array}{l}2018 \\
\text { (p) }\end{array}$ & $6.918 .891,0$ & $93.037 .062,0$ & $7.606 .055,0$ & $107.562 .008,0$ & $6.562 .617,0$ & $62.917 .906,0$ & $2.389 .994,0$ & $71.870 .517,0$ & $-6,4$ & 2,0 & 4,9 & 1,3 \\
\hline
\end{tabular}

Fuente: Banco Central del Ecuador (2019) 


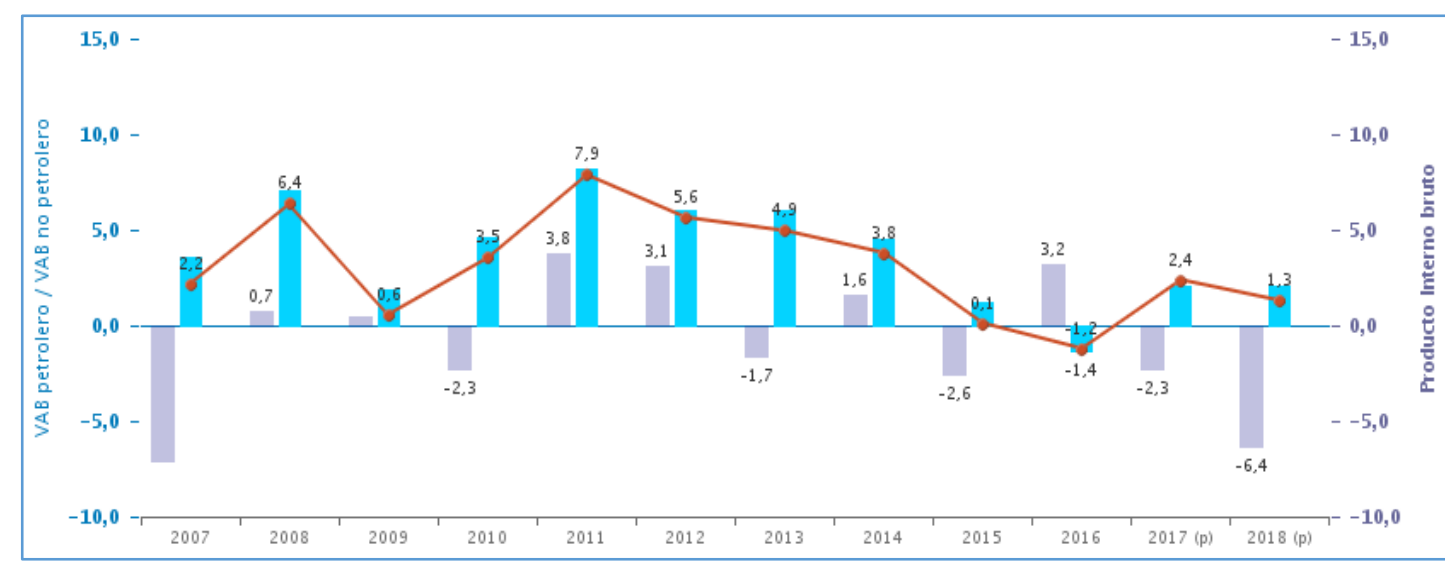

Valor Agregado no Petrolero

Valor Agregado PIB

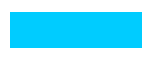
Petrolero
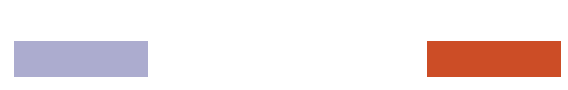

Figura 3. Valor Agregado Bruto Petrolero y no Petrolero del Ecuador y Tasas de Variación anual (en \%). Período 2007-2018.

Fuente: Banco Central del Ecuador (2019).

Como se puede evidenciar en la Tabla 2 y Figura 3, la evolución del VAB Petrolero dentro del período 2007 - 2018 ha venido acompañada de múltiples fluctuaciones asociadas a la disminución en la extracción de petróleo crudo en el contexto del mercado internacional. Pese a la notable recuperación que el país experimentó entre 2007 y 2008, donde la tasa de variación se ubicó por encima del $0.7 \%$, luego, sin embargo, sintió los efectos de la recesión del 2008, con una caída de alrededor de USD 3.878 millones. Entre 2010 y 2015 se refleja un crecimiento sostenido en sus cifras, bordeando valores de USD 10.902 millones. El período 2016 - 2018 se encuentra marcado por la guerra de precios desatada entre la OPEP y los productores norteamericanos, que afectó abruptamente el mercado de crudo y terminó incidiendo en una colosal caída del VAB petrolero, cerrando el 2018 con una cifra aproximadamente de USD 6.918 millones.

El VAB No Petrolero, por otra parte, refleja una evolución aproximada del $2 \%$ entre el 2017 y 2018, y una tasa de variación global período 2007 - 2018 de 3.8\%, que como indica el Banco Central del Ecuador (2018) "se explica por un mayor dinamismo de industrias como: acuicultura; enseñanza y servicios sociales; alojamiento; suministros; comercio y agricultura", principalmente. De la misma manera, el PIB muestra caídas importantes en el 2008 y 2016, coincidente a las contracciones económicas ligadas a la crisis de los commodities, pero a su vez, un crecimiento importante, pasando de ubicarse nominalmente de los USD 51.007millones en 2007 a USD 107.562 millones en 2018. 
Tabla 3. Valor Agregado Bruto provincial del Ecuador, región Costa. Periodo 2007 -2018. (en USD)

\begin{tabular}{|c|c|c|c|c|c|c|c|c|c|}
\hline \multirow{3}{*}{ A ÑO } & \multicolumn{9}{|c|}{ Valor Agregado Bruto Nacional Período 2007 - 2018} \\
\hline & \multicolumn{9}{|c|}{ Región Costa } \\
\hline & \multicolumn{2}{|c|}{ Esmeraldas } & \multicolumn{2}{|c|}{ Manabí } & \multirow{2}{*}{$\begin{array}{c}\text { Los Ríos } \\
\$ 1,631,611\end{array}$} & \multirow{2}{*}{$\begin{array}{c}\text { Guayas } \\
\$ 12,733,775\end{array}$} & Santa Elena & \multicolumn{2}{|r|}{ El Oro } \\
\hline 2007 & $\$$ & $1,548,215$ & $\$$ & $2,737,602$ & & & $\$ \quad-$ & $\$$ & $1,532,516$ \\
\hline 2008 & $\$$ & $1,769,914$ & $\$$ & $3,373,794$ & $\$ 1,960,898$ & $\$ 14,228,422$ & $\$ 1,147,822$ & $\$$ & $1,912,579$ \\
\hline 2009 & $\$$ & $1,847,345$ & $\$$ & $3,491,303$ & $\$ 2,145,279$ & $\$ 15,131,399$ & $\$ 1,203,359$ & $\$$ & $2,088,056$ \\
\hline 2010 & $\$$ & $1,900,158$ & $\$$ & $3,748,862$ & $\$ 2,413,545$ & $\$ 16,497,888$ & $\$ 1,141,082$ & $\$$ & $2,125,002$ \\
\hline 2011 & $\$$ & $2,142,741$ & $\$$ & $4,542,663$ & $\$ 2,704,536$ & $\$ 18,354,822$ & $\$ 1,042,099$ & $\$$ & $2,510,806$ \\
\hline 2012 & $\$$ & $1,971,092$ & $\$$ & $4,879,159$ & $\$ 2,744,965$ & $\$ 20,437,366$ & $\$ 1,293,447$ & $\$$ & $2,857,237$ \\
\hline 2013 & $\$$ & $1,977,460$ & $\$$ & $5,267,038$ & $\$ 2,997,209$ & $\$ 22,763,860$ & $\$ 1,219,751$ & $\$$ & $3,036,097$ \\
\hline 2014 & $\$$ & $2,144,296$ & $\$$ & $5,462,859$ & $\$ 3,319,032$ & $\$ 24,749,658$ & $\$ 1,284,007$ & $\$$ & $3,331,275$ \\
\hline 2015 & $\$$ & $2,518,870$ & $\$$ & $5,599,683$ & $\$ 3,555,661$ & $\$ 24,409,213$ & $\$ 1,253,875$ & $\$$ & $3,293,032$ \\
\hline 2016 & $\$$ & $2,946,460$ & $\$$ & $6,046,460$ & $\$ 3,545,911$ & $\$ 25,466,251$ & $\$ 1,154,788$ & $\$$ & $3,173,793$ \\
\hline 2017 & $\$$ & $3,047,275$ & $\$$ & $6,212,365$ & $\$ 3,675,429$ & $\$ 25,815,766$ & $\$ 1,358,838$ & $\$$ & $3,467,436$ \\
\hline 2018 & $\$$ & $3,086,832$ & $\$$ & $6,062,625$ & $\$ 3,557,099$ & $\$ 26,637,131$ & $\$ 1,547,883$ & $\$$ & $3,570,852$ \\
\hline
\end{tabular}

Fuente: Banco Central del Ecuador (2019). 
Tabla 4. Valor Agregado Bruto provincial del Ecuador, región Sierra. Periodo 2007 -2018. (en USD)

\begin{tabular}{|c|c|c|c|c|c|c|c|c|c|c|c|c|c|}
\hline \multirow[b]{3}{*}{ A ÑO } & \multicolumn{13}{|c|}{ Valor Agregado Bruto Nacional Período 2007 - 2018} \\
\hline & & \multicolumn{12}{|c|}{ Región Sierra } \\
\hline & & Carchi & Imbabura & Pichincha & $\begin{array}{c}\text { Santo } \\
\text { Domingo }\end{array}$ & Cotopaxi & Tungurahua & & olívar & Chimborazo & Cañar & Azuay & Loja \\
\hline 2007 & & 342,390 & $\$ 923,304$ & $\$ 12,373,553$ & $\$ \quad-$ & $\begin{array}{c}\$ \\
843,035\end{array}$ & $\$ 1,301,034$ & $\$$ & 286,972 & $\$ 840,618$ & $\$ 536,087$ & $\$ 2,573,009$ & $\$ 992,948$ \\
\hline 2008 & & 391,668 & $\$ 1,026,131$ & $\$ 13,740,114$ & $\$ 1,009,329$ & $\begin{array}{c}\$ \\
1,000,086\end{array}$ & $\$ 1,509,677$ & $\$$ & 322,983 & $\$ 967,059$ & $\$ 604,153$ & $\$ 2,887,742$ & $\$ 1,162,973$ \\
\hline 2009 & $\$$ & 450,458 & $\$ 1,136,803$ & $\$ 14,414,168$ & $\$ 1,046,317$ & $\begin{array}{c}\$ \\
1,092,561 \\
\end{array}$ & $\$ 1,696,358$ & $\$$ & 369,645 & $\$ 1,053,989$ & $\$ \quad 669,826$ & $\$ 3,054,408$ & $\$ 1,255,653$ \\
\hline 2010 & & 483,136 & $\$ 1,328,059$ & $\$ 16,449,770$ & $\$ 1,109,313$ & $\begin{array}{c}\$ \\
1,237,680\end{array}$ & $\$ 1,792,863$ & $\$$ & 376,957 & $\$ 1,125,403$ & $\$ \quad 688,416$ & $\$ 3,353,436$ & $\$ 1,296,101$ \\
\hline 2011 & & 527,391 & $\$ 1,530,583$ & $\$ 18,292,542$ & $\$ 1,264,977$ & $\begin{array}{c}\$ \\
1,346,607\end{array}$ & $\$ 2,029,842$ & $\$$ & 416,019 & $\$ 1,287,639$ & $\$ \quad 806,927$ & $\$ 3,727,619$ & $\$ 1,495,633$ \\
\hline 2012 & & 573,726 & $\$ 1,703,467$ & $\$ 20,662,585$ & $\$ 1,596,313$ & $\begin{array}{c}\$ \\
1,456,535\end{array}$ & $\$ 2,271,712$ & $\$$ & 427,905 & $\$ 1,324,404$ & $\$ \quad 835,265$ & $\$ 3,944,848$ & $\$ 1,598,213$ \\
\hline 2013 & & 571,991 & $\$ 1,751,037$ & $\$ 22,956,885$ & $\$ 1,587,044$ & $\begin{array}{c}\$ \\
1,523,687 \\
\end{array}$ & $\$ 2,375,376$ & $\$$ & 454,001 & $\$ 1,420,254$ & $\$ 932,562$ & $\$ 4,222,873$ & $\$ 1,685,540$ \\
\hline 2014 & & 636,644 & $\$ 1,867,284$ & $\$ 25,865,822$ & $\$ 1,647,361$ & $\begin{array}{c}\$ \\
1,586,461\end{array}$ & $\$ 2,524,414$ & $\$$ & 483,386 & $\$ 1,626,191$ & $\$ 938,944$ & $\$ 4,432,976$ & $\$ 1,685,620$ \\
\hline 2015 & & 684,754 & $\$ 1,877,162$ & $\$ 25,312,810$ & $\$ 1,799,660$ & $\begin{array}{c}\$ \\
1,731,339\end{array}$ & $\$ 2,757,000$ & $\$$ & 554,763 & $\$ 1,859,165$ & $\$ 1,025,294$ & $\$ 4,765,706$ & $\$ 1,837,641$ \\
\hline 2016 & & 663,569 & $\$ 1,811,870$ & $\$ 25,650,241$ & $\$ 1,841,475$ & $\begin{array}{c}\$ \\
1,699,624 \\
\end{array}$ & $\$ 2,682,274$ & $\$$ & 580,028 & $\$ 1,974,581$ & $\$ 1,024,577$ & $\$ 4,782,811$ & $\$ 1,799,345$ \\
\hline 2017 & & 662,901 & $\$ 1,846,117$ & $\$ 26,406,871$ & $\$ 1,886,213$ & $\begin{array}{c}\$ \\
1,837,791 \\
\end{array}$ & $\$ 2,857,483$ & $\$$ & 634,678 & $\$ 1,900,010$ & $\$ 1,124,016$ & $\$ 5,013,920$ & $\$ 1,751,490$ \\
\hline 2018 & & 663,975 & $\$ 1,930,397$ & $\$ 26,571,645$ & $\$ 2,037,680$ & $\begin{array}{c}\$ \\
1,952,861\end{array}$ & $\$ 2,866,045$ & $\$$ & 638,971 & $\$ 1,854,455$ & $\$ 1,065,192$ & $\$ 5,343,370$ & $\$ 1,774,545$ \\
\hline
\end{tabular}

Fuente: Banco Central del Ecuador (2019). 
Tabla 5. Valor Agregado Bruto provincial del Ecuador, Región Amazónica. Periodo 2007 -2018. (en USD)

\begin{tabular}{|c|c|c|c|c|c|c|c|c|c|c|c|c|c|}
\hline \multirow{4}{*}{$\begin{array}{l}\text { A ÑO } \\
2007 \\
\end{array}$} & \multicolumn{11}{|c|}{ Valor Agregado Bruto Nacional Período 2007 - 2018} & \multirow{3}{*}{\multicolumn{2}{|c|}{ Total Nacional }} \\
\hline & \multicolumn{9}{|c|}{ Región Amazónica } & \multirow{2}{*}{\multicolumn{2}{|c|}{ Islas Galápagos }} & & \\
\hline & \multirow{2}{*}{$\begin{array}{l}\text { Sucumbíos } \\
\$ 1,919,074\end{array}$} & & \multirow{2}{*}{$\begin{array}{c}\text { Orellana } \\
\$ 3,947,584\end{array}$} & Pastaza & \multicolumn{2}{|c|}{$\begin{array}{l}\text { Morona } \\
\text { Santiago }\end{array}$} & \multicolumn{2}{|c|}{$\begin{array}{l}\text { Zamora } \\
\text { Chinchipe }\end{array}$} & & & & \\
\hline & & $\$$ & 160,276 & & $\$ 783,553$ & $\$$ & 193,089 & $\$$ & 141,669 & $\$$ & 168,989 & $\$$ & $48,510,903$ \\
\hline 2008 & $\$ 2,532,197$ & $\$$ & 195,240 & $\$ 6,104,988$ & $\$ 1,077,145$ & $\$$ & 250,684 & $\$$ & 179,487 & $\$$ & 195,816 & $\$$ & $59,550,902$ \\
\hline 2009 & $\$ 1,725,078$ & $\$$ & 223,654 & $\$ 3,565,014$ & 659,558 & $\$$ & 271,535 & $\$$ & 215,669 & $\$$ & 205,841 & $\$$ & $59,013,276$ \\
\hline 2010 & $\$ 4,258,471$ & $\$$ & 248,915 & $\$ 3,468,477$ & $\$ 759,702$ & $\$$ & 300,974 & $\$$ & 210,861 & $\$$ & 184,389 & $\$$ & $66,499,460$ \\
\hline 2011 & $\$ 3,470,682$ & $\$$ & 294,303 & $\$ 6,808,895$ & $\$ 1,134,771$ & $\$$ & 374,366 & $\$$ & 247,478 & $\$$ & 182,275 & $\$$ & $76,536,215$ \\
\hline 2012 & $\$ 3,066,769$ & $\$$ & 303,503 & $\$ 8,034,596$ & $\$ 1,042,881$ & $\$$ & 385,578 & $\$$ & 256,289 & $\$$ & 188,316 & $\$$ & $83,856,171$ \\
\hline 2013 & $\$ 3,186,625$ & $\$$ & 309,352 & $\$ 8,451,545$ & $\$ 1,032,771$ & $\$$ & 378,474 & $\$$ & 244,086 & $\$$ & 188,344 & $\$$ & $90,533,862$ \\
\hline 2014 & $\$ 2,755,772$ & $\$$ & 333,449 & $\$ 8,367,418$ & $\$ 981,797$ & $\$$ & 406,380 & $\$$ & 257,160 & $\$$ & 206,531 & $\$$ & $96,894,741$ \\
\hline 2015 & $\$ 1,810,651$ & $\$$ & 367,731 & $\$ 3,466,041$ & 613,011 & $\$$ & 452,884 & $\$$ & 289,567 & $\$$ & 206,992 & $\$$ & $92,042,505$ \\
\hline 2016 & $\$ 1,592,969$ & $\$$ & 378,685 & $\$ 2,695,345$ & 546,685 & $\$$ & 460,114 & $\$$ & 292,668 & $\$$ & 227,764 & $\$$ & $93,038,286$ \\
\hline 2017 & $\$ 1,508,821$ & $\$$ & 479,632 & $\$ 3,935,351$ & 634,614 & $\$$ & 505,543 & $\$$ & 277,482 & $\$$ & 242,691 & $\$$ & $97,082,733$ \\
\hline 2018 & $\$ 1,888,026$ & $\$$ & 463,368 & $\$ 4,664,330$ & 713,844 & $\$$ & 507,871 & $\$$ & 302,421 & $\$$ & 254,534 & $\$$ & $99,955,953$ \\
\hline
\end{tabular}

Fuente: Banco Central del Ecuador (2019). 


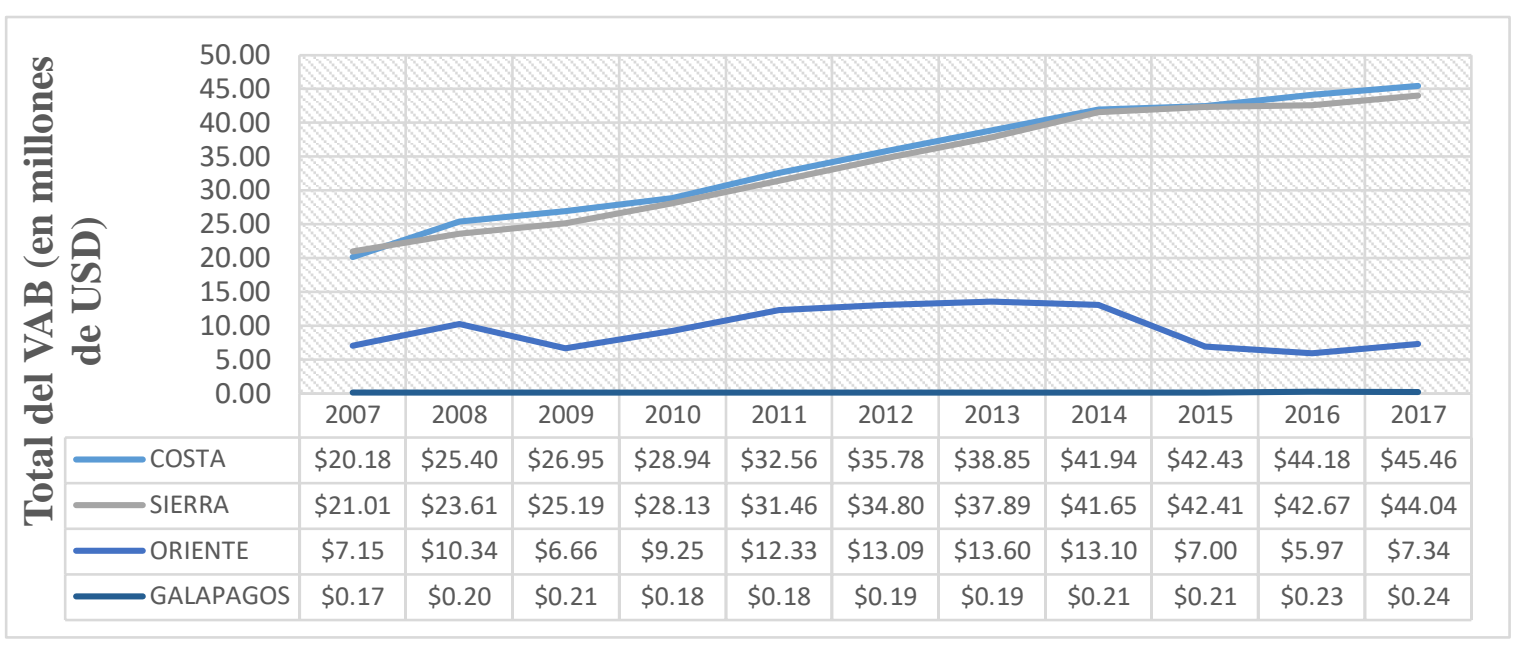

Figura 4. Crecimiento promedio al valor agregado bruto regional. Periodo 2007 -2017. Fuente: Banco Central del Ecuador (2019).

La Figura 4, refleja el crecimiento promedio al VAB por regiones y el aporte total al VAB nacional. Se aprecia que la Región Costa es la que representa una mayor participación al VAB, desde el año 2007 hasta el 2017, con una tasa promedio de crecimiento del 8,64\% y aporte del 44,31\% al VAB nacional; mientras que la Región Sierra crece para el periodo anteriormente señalado a una tasa promedio del $7,76 \%$ y aporta el $43.17 \%$ al VAB nacional, siendo la segunda región con mayor aporte en el país. La Región Oriente tiene una tasa promedio de crecimiento del 4,94\% y aporta el 12,25\% al VAB nacional, seguido de la Región Insular o Galápagos que es la que menor aporte representa, con una tasa de crecimiento del $3,92 \%$ y un aporte del $0,25 \%$ al VAB nacional. 


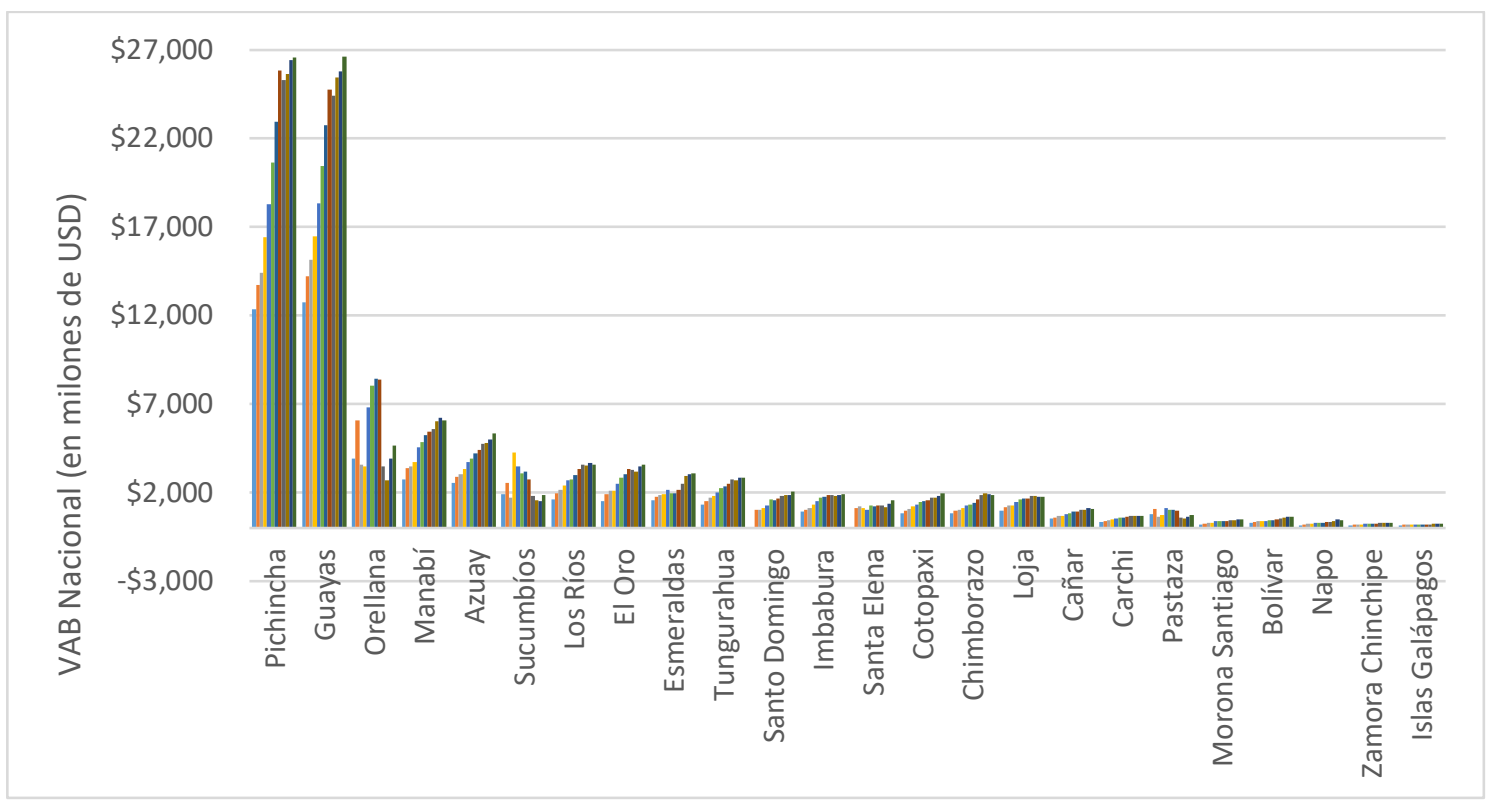

Figura 5. Valor Agregado Bruto Nacional. Periodo 2007 -2018.

Fuente: Banco Central del Ecuador (2019).

Los datos reflejados en la Figura 5 ponen de manifiesto la gradiente centro - periferia históricamente existente en nuestro país, donde las provincias de Pichincha y Guayas concentran el $44 \%$ de la población del país y un 54\% del PIB nacional. Por citar un caso en específico, en 2007 agruparon USD 12.733 y USD 12.373 millones respectivamente, año en el que el VAB Nacional Total alcanzaría los USD 48.510 .903 millones, lo que se traduce en un aporte en conjunto del $51.76 \%$ frente a las otras 20 provincias restantes. Este fenómeno es muy recurrente y se reitera en cada uno de los años del período analizado.

Si centramos el análisis referente al aporte medido por regiones, se aprecia como resultado que la Región Costa, tomando como ejemplo el año 2018, contribuye con el $44.48 \%$ del VAB, se evidencia a la Región Sierra con un aporte del $46.72 \%$, y en la Región Amazónica con el 8.54\% y la Región Insular con el 0.25\%. Dentro de esta perspectiva, es imperativo resaltar el aporte mayoritario que en la Región Costa realizan en promedio entre 2007 y 2018 las provincias de Guayas con el 25.66\% y Manabí con el $5.96 \%$, por su parte en la Región Sierra, Pichincha en promedio en el mismo período mantiene una contribución del 25.81\% y Azuay con el 4.99\%. En la Amazonía, Orellana destaca con un aporte promedio mayoritario del 6.59\% y Sucumbíos con el 3.08\%.

La información presentada en la Tabla 3, 4 y 5, es una muestra palpable de la desigual contribución de las provincias del país como consecuencia directa del inequitativo desarrollo económico que éstas experimentan. Especial preocupación reflejan las provincias del centro y sur de la Amazonía y de la sierra-norte, con un minúsculo aporte al Valor Agregado Bruto Nacional del $6.18 \%$ y $2.19 \%$ respectivamente y una evolución relativamente pobre en su crecimiento durante el período 2007 - 2018 analizado.

Las cuentas regionales permiten cuantificar y conocer la estructura económica y la especialización productiva de cada provincia y cantón del país, como una extensión de la Contabilidad Nacional. Son herramientas muy valiosas para el análisis de los desafíos de la economía local y nacional. 


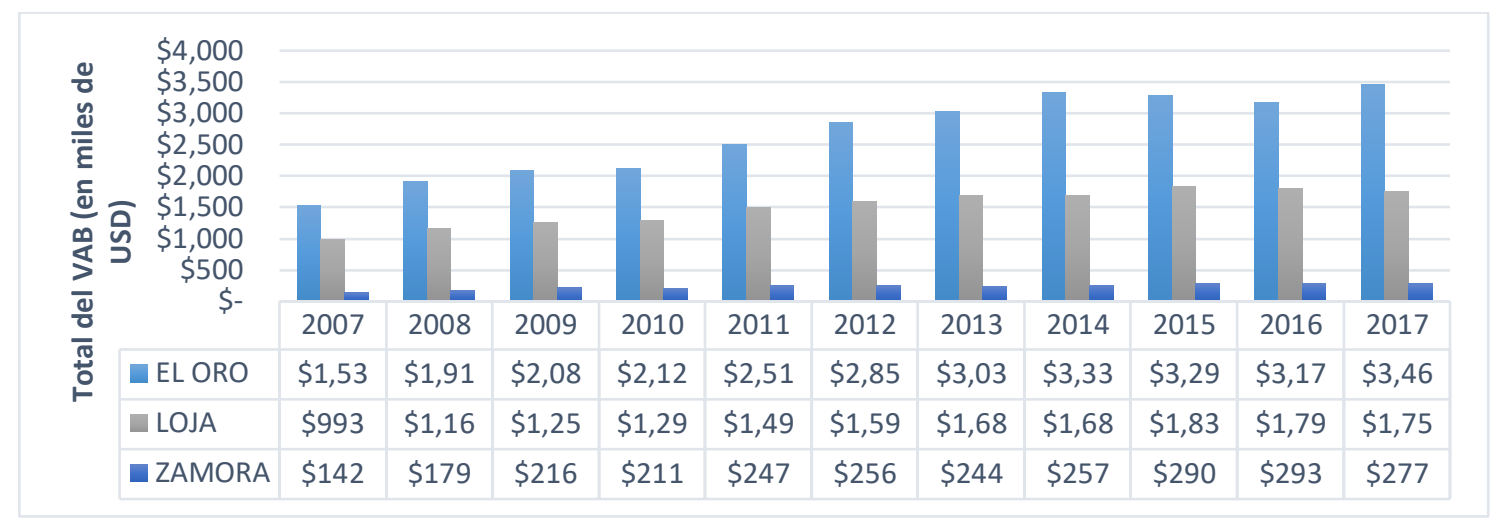

Figura 6. Zona 7 - VAB total Zona 7. Periodo 2007 -2017

Fuente: Banco Central del Ecuador (2019).

Las zonas de planificación de Ecuador son entes de organización administrativa conformados por provincias conjuntas o distritos metropolitanos que tienen el fin de desconcentrar las actividades administrativas del Estado ecuatoriano. La zona 7 - Sur del Ecuador, está conformada por las provincias de El Oro, Loja y Zamora Chinchipe.

En la Figura 6 se evidencia la evolución del VAB período 2007 - 2017 en la Zona Administrativa 7 compuesta por las provincias de El Oro, Loja y Zamora Chinchipe. El crecimiento en este período demuestra un claro contraste entre sus componentes, mientras que la provincia de El Oro pasó de aportar USD 1.53 millones correspondiente al $57.46 \%$ al total del VAB en 2007 a USD 3.46 millones con el 63.09\% en 2017, Loja vio reducida su participación cayendo de USD 992 mil correspondiente al 37.23\% en 2007 a USD 1.75 millones con el $31.87 \%$ de aporte en 2017 . Pese a esto, ambas provincias presentan una tasa promedio de crecimiento anual del $0.08 \%$ y $0.05 \%$ respectivamente. El panorama de Zamora Chinchipe es en proporción bastante dispar a las otras dos provincias analizadas. Su porcentaje de participación en la última década incluso se ha reducido, pasando del $5.31 \%$ con el aporte de USD $141 \mathrm{mil}$ en el 2007 a un 5.05\% de USD 277 mil en 2017. El crecimiento anual promedio bordea el $5.39 \%$ y es un reflejo de su debilitada estructura productiva y la diseminada concentración poblacional.

La provincia de El Oro es una de las más reconocidas e importantes de la región de la Costa, es una de los núcleos administrativos, comerciales, financieros, de mayor relevancia y aporte económico en el país. Entre las principales actividades económicas que propician la prosperidad de esta provincia está la producción agrícola, el comercio a través de Puerto Bolívar, la ganadería y la industria. 


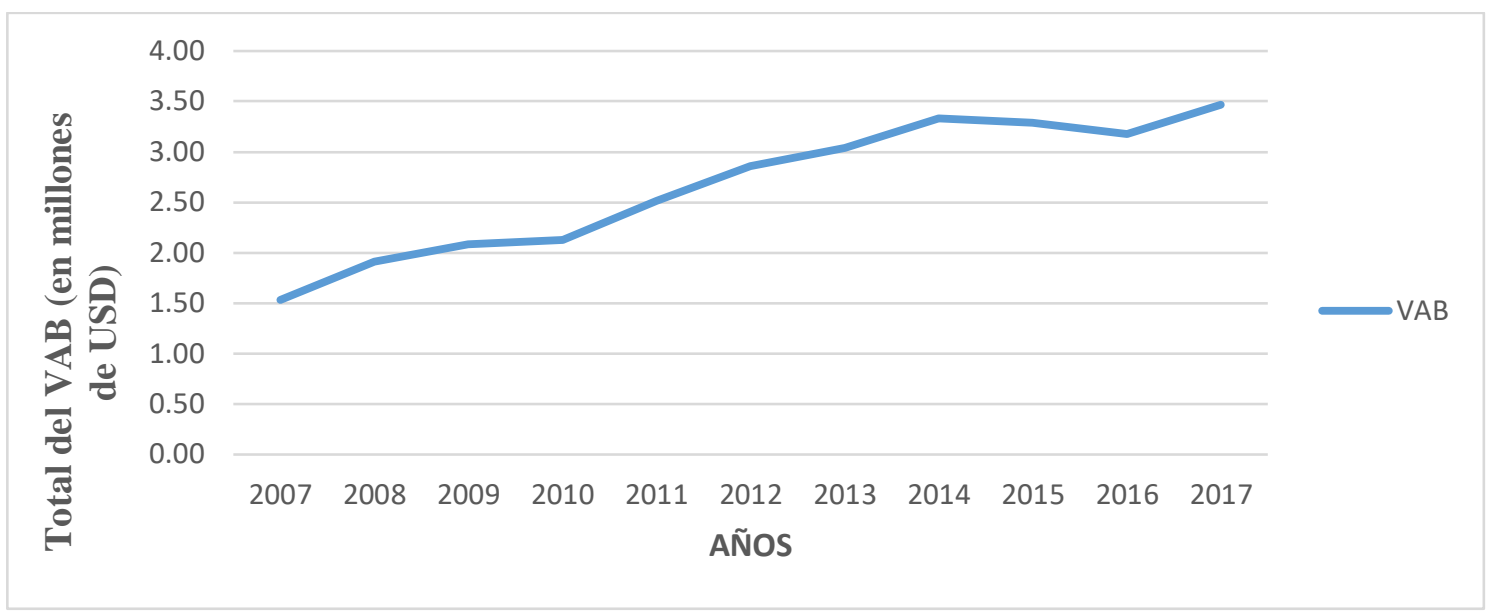

Figura 7. Evolución del VAB de la Provincia de El Oro 2007 - 2017

Fuente: Banco Central del Ecuador (2019).

En la Figura 7, la provincia de El Oro es una de las más ricas del Ecuador, donde se enfatiza la producción de bienes del sector primario como eje principal para el crecimiento del VAB a nivel nacional, otorgándole a la región Costa ventajas competitivas al compararlas con otras provincias del país (Capa, Sotomayor, \& Vega, 2018). Para el año 2017 el VAB alcanzó alrededor de los USD 3.400 millones. El sector primario de la provincia de El Oro genera crecimientos constantes a nivel nacional, por tal razón la provincia contribuye al VAB representando el 3,32\%, destacándose diversas actividades productivas, entre las principales se menciona la producción primaria, por la cual se caracteriza la provincia, seguido del comercio, entre otros (Ollague, y otros, 2019).

Los sectores productivos se clasifican en sector primario, secundario y terciario, son actividades que producen bienes tangibles (son medibles y cuantificables) e intangibles. El desarrollo relativo de cada sector de la economía obedece a muchos factores intrínsecos y extrínsecos de las mismas. Ahora nos vamos a centrar en el análisis por sectores de la provincia de El Oro. Es notorio el débil aporte a la economía provincial del sector secundario.

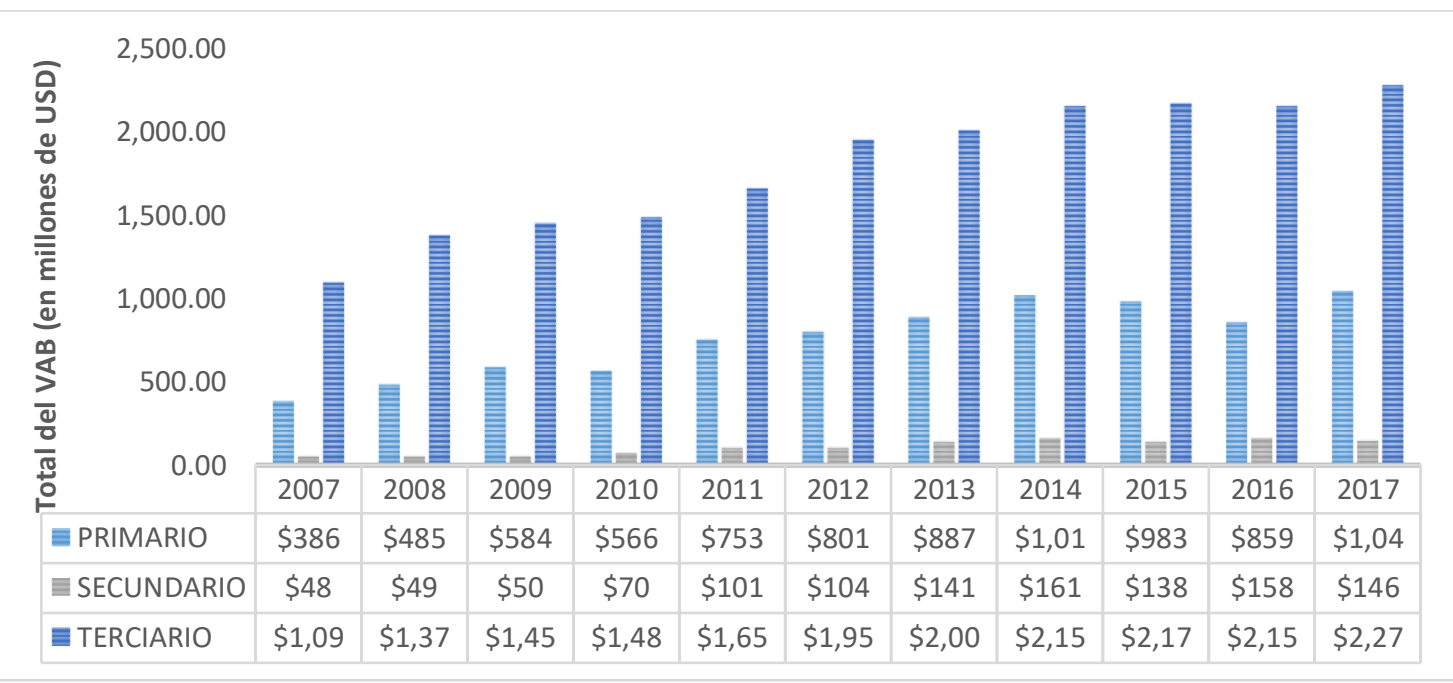

Figura 8: Total del VAB de la provincia de El Oro y sus sectores. Periodo 2007 -2017 (en millones de USD).

Fuente: Banco Central del Ecuador (2019). 
La Figura 8 refleja la evolución del VAB de la provincia de El Oro subdivido en los sectores primario, secundario y terciario durante el período 2007 - 2017. Es evidente el crecimiento sostenido y generalizado de los sectores Primario (siendo el sector agrícola y acuícola protagonistas) y el sector Terciario (servicios y turismo los principales). Estos sectores tienen una tasa de crecimiento anual promedio del $0.09 \%$ y $0.07 \%$ respectivamente. Por su parte, el sector secundario tiene un papel mucho más reducido dentro del VAB provincial, su participación promedio en el período 2007 - 2017 del $3.98 \%$, frente al $28.52 \%$ del sector Primario y del $67.50 \%$ del Terciario. Esto se ve reflejado en la realidad productiva de la provincia, constituyéndose mayoritariamente como comercializadora de productos agrícolas, acuícolas y mineros; además del fortalecimiento del sector servicios y la minúscula presencia de industrias transformadoras de materias primas.

\section{Conclusión}

La presente investigación ha permitido apreciar la importancia que tiene el VAB en los niveles de crecimiento y desarrollo económico de Ecuador. Es evidente que, si una nación desea vencer la pobreza, deberá diseñar y ejecutar políticas económicas tendentes a fortalecer la producción nacional. En este sentido el fomento a la inversión y estabilidad jurídica, política y económica, así como un entramado legal (laboral y tributario), juegan un papel fundamental para el logro de ese objetivo.

Por otra parte, se advierte la relevancia del sector primario como eje rector de la producción nacional, así como la dependencia de la producción por el petróleo. Por lo tanto, resulta necesario analizar la estructura de la matriz productiva del país y direccionarla hacia la generación de bienes de valor agregado y servicios especializados, dada la cantidad de recursos existentes en Ecuador, a sabiendas que el petróleo es un recurso no renovable y las reservas se agotarán en algún momento. La economía debe planificarse en el largo plazo.

En tercer lugar, podemos valorar la extraordinaria relevancia que tienen las provincias de Pichicha y Guayas en el contexto nacional. Poco más del 50\% del total del VAB nace de estas provincias, lo cual configura una relación centro periferia, donde el centro de la actividad económica lo tienen las provincias de la Costa y Sierra central (Pichincha y Guayas en primer lugar) y la periferia constituida por las seis provincias del Oriente, las cuales no concentran más del 6,5\% del total del VAB nacional.

Por su parte la provincia de El Oro representa algo más del 5\% del total del VAB nacional y dentro de la Región 7 es la que más representatividad posee. Esta provincia, al igual que el país, se caracteriza por la producción de bienes primarios orientados a la exportación; el banano, cacao, camarón y explotación de oro se constituyen en los principales referentes de producción.

Surge entonces la necesidad de estudiar a profundidad la posibilidad de que el país explore y defina alternativas para su desarrollo productivo. Si el deseo común es crecer económicamente y vencer la pobreza, se hace imprescindible agregar valor a la producción, mejorar la estructura productiva, buscar mercados y exportar bienes sobre los que se tenga ventajas competitivas y comparativas con respecto a otros países. 


\section{Referencias}

Aravena , C., \& Fuentes, J. A. (2013). El desempeño mediocre de la productividad laboral en América Latina: una interpretación neoclásica. Santiago de Chile: CEPAL. Obtenido de https://repositorio.cepal.org/bitstream/handle/11362/5373/1/LCL3725.pdf

Brito, L., Sotomayor, G., \& Apolo, J. (2019). Análisis y perspectivas del valor agregado bruto en la economía ecuatoriana. X-Pedientes Económicos, 3(5), 17-36. Obtenido de https://ojs.supercias.gob.ec/index.php/X-pedientes_Economicos/article/view/80/23

Capa, L., Sotomayor, J., \& Vega, F. (2018). La Provincia de El Oro algunas consideraciones de los sectores productivos y empresariales. Machala: UTMACH. Obtenido de http://repositorio.utmachala.edu.ec/bitstream/48000/12490/1/LaProvinciaDeEloroAlgun asConsideracionesDelSectorProductivoYEmpresarial.pdf

Cruz, M., \& Polanco, M. (2014). El sector primario y el estancamiento económico en México. Problemas del Desarrollo, 45(178), 9-33. Obtenido de http://www.revistas.unam.mx/index.php/pde/article/view/47833

García, D., \& Ochoa, S. (2018). Relación entre crecimiento económico y medio ambiente en Ecuador a nivel provincial (Promedio 2010-2015). REVISTA CIENCIA $Y$ TECNOLOGÍA, 6(2), 99-112. Obtenido de https://revistas.proeditio.com/REVISTAMAZONICA/article/view/2328/2387

Gómez Luna, E., Navas, D., Aponte Mayor, G., \& Betancourt Buitrago, L. (2014). Metodología para la revisión bibliográfica y la gestión de información de temas científicos, a través de su estructuración y sistematización. revista de la Facultad de Minas, 81(184), 158163. Obtenido de file:///C:/Users/usuario/Downloads/DialnetMetodologiaParaLaRevisionBibliograficaYLaGestionDe-4717293.pdf

Guerra, C., \& González, I. (2015). La relación dinámica del valor agregado bruto, la producción mercantil y el gasto material.Su importancia para la toma de decisiones. Ecnomía y Desarrollo, 151(1), 118-131. Obtenido de https://www.redalyc.org/articulo.oa?id=425541212009

Ollague, J., Capa, L., Novillo , E., Sánchez, T., Sánchez Liana, \& García, M. (2019). Variables sociales, económicas y productivas como referente de posicionamiento nacional de la provincia de El Oro, Ecuador. Espacios, 40(37), 13-29. Obtenido de https://www.revistaespacios.com/a19v40n37/a19v40n37p13.pdf

Pereira, S., Apunte, R., \& Pulgar, M. E. (2018). RELACIÓN ENTRE EL DESEMPEÑO DE LAS PYME Y EL VALOR AGREGADO BRUTO. CienciAmérica, 7(1), 98-108. Obtenido de http://cienciamerica.uti.edu.ec/openjournal/index.php/uti/article/view/156/148

Tancara Q., C. (1993). LA INVESTIGACION DOCUMENTAL. Temas Sociales, 91-106. Obtenido de http://www.scielo.org.bo/scielo.php?script=sci_arttext\&pid=S004029151993000100008 
Vega, F., Brito, L., Apolo, N., \& Sotomayor, J. (2020). Influencia de la recaudación fiscal en el valor agregado bruto de los cantones de la provincia de El Oro (Ecuador), para el periodo 2007-2017. ESPACIOS, 41(15), 15-27. Obtenido de https://www.revistaespacios.com/a20v41n15/20411515.html

Zamora, R., \& Coello, G. (2015). El valor agregado bruto (VAB) del comercio en la provincia de Tungurahua. Revista de Coyuntura. Observatorio Económico y Social de Tungurahua, 2, 7-10. Obtenido de https://fca.uta.edu.ec/v3.2/servicios/observatorio/revistas/revista2.pdf 